\title{
On the Equivalence of Two Security Notions for Hierarchical Key Assignment Schemes in the Unconditional Setting
}

\author{
Massimo Cafaro, Senior Member, IEEE, Roberto Civino, and Barbara Masucci
}

\begin{abstract}
The access control problem in a hierarchy can be solved by using a hierarchical key assignment scheme, where each class is assigned an encryption key and some private information. A formal security analysis for hierarchical key assignment schemes has been traditionally considered in two different settings, i.e., the unconditionally secure and the computationally secure setting, and with respect to two different notions: security against key recovery (KR-security) and security with respect to key indistinguishability (KI-security), with the latter notion being cryptographically stronger. Recently, Freire, Paterson and Poettering proposed strong key indistinguishability (SKI-security) as a new security notion in the computationally secure setting, arguing that SKI-security is strictly stronger than $\mathrm{KI}$-security in such a setting. In this paper we consider the unconditionally secure setting for hierarchical key assignment schemes. In such a setting the security of the schemes is not based on specific unproven computational assumptions, i.e., it relies on the theoretical impossibility of breaking them, despite the computational power of an adversary coalition. We prove that, in this setting, SKI-security is not stronger than KI-security, i.e., the two notions are fully equivalent from an information-theoretic point of view.
\end{abstract}

Index Terms-Access controls, Information flow controls, Hierarchical design, Data dependencies, Coding and Information Theory

\section{INTRODUCTION}

T $\mathrm{N}$ the access control problem, we are concerned with the problem of ensuring that only authorized users of a computer system are entitled to access sensitive information, according to access control policies that organize users in a hierarchy of disjoint classes, called security classes. The main reason for a hierarchy is the need to assign different privileges and right sto users, according to their role, competencies and responsibilities.

Hierarchies model and reflect many real world cases. A classical example is a hospital, in which doctors are allowed accessing data concerning their patients, but other people down in the hierarchy may access this information only partially (for instance, external researchers may be given access to anonymized data for clinical studies) or not at all (e.g., paramedics may be denied access). Many different instances of the access control problem commonly arise in government agencies, in the military realm where classified documents can be accessed by selected users on the

- M. Cafaro is with the Department of Engineering for Innovation, University of Salento, Lecce 73100, Italy

E-mail:massimo.cafaro@unisalento.it

- R. Civino is with the Department of Mathematics and Physics, University of Salento, Lecce 73100, Italy

E-mail: roberto.civino@studenti.unisalento.it

- B. Masucci is with the Department of Computer Science, University of Salerno, Fisciano 84084, Italy

E-mail:masucci@dia.unisa.it basis of their security clearance level, etc.

In a hierarchical key assignment scheme, an encryption key and some private information are assigned to each class in the hierarchy. The encryption key protects each class data by means of a symmetric crypto-system, whereas private information allows each class to compute the keys assigned to all of the classes lower down in the hierarchy. This assignment is carried out by a central authority, the so-called Trusted Authority (TA), which is active only in the initial distribution phase.

Hierarchical key assignment schemes were first proposed in the seminal paper of Akl and Taylor [1], and improved in order to achieve better performances or to allow hierarchies in which classes can be dynamically inserted or deleted [2], [15], [17], [18], [19], [21], [22]. Related work includes further research focusing on more general access control policies [10], [20], [29]. Even though a large number of schemes had been proposed, the lack of a formal security proof for many of them led to an initial analysis of the security properties of these schemes, which confirmed that, indeed, those schemes are insecure against collusive attacks in which a coalition of users tries attacking a class up in the hierarchy [5], [23], [28], [29]. A useful taxonomy and evaluation of several schemes appeared in the literature can be found in [8]. A related branch of research, focusing on the design of hierarchical key assignment schemes with time-dependent keys, called time-bound schemes, was started by Tzeng [25]. However, Tzeng's scheme was shown later to 
be insecure against collusive attacks [31]. Additional efforts include [6], [16], [28], but none of these schemes is secure against collusive attacks as well [3], [11], [24], [30]. New time-bound schemes, derived from the AklTaylor scheme, were given by Wang and Laih [27] and Tzeng [26] and further analyzed in [9].

The most used approach to hierarchical key assignment schemes is based on unproven specific computational assumptions. In this computational setting, a hierarchical key assignment scheme is provably-secure under a complexity assumption if the existence of an adversary $A$ breaking the scheme is equivalent to the existence of an adversary $B$ breaking the computational assumption. The usual method of construction of $B$ uses the adversary $A$ as a black-box. The need for formal security requirements in the computational setting was first addressed in [2] by Atallah et al., who proposed two different security notions for hierarchical key assignment schemes: security against key recovery (KR-security) and security with respect to key indistinguishability (KI-security). KR-security corresponds to the requirement that an adversary is not able to compute a key that it should not have access to. On the other hand, KI-security formalizes the requirement that the adversary is not able to learn any information about a key that it should not have access to, i.e., it is not able to distinguish it from a random string having the same length.

Recently, Freire et al. [14] proposed a new security notion for hierarchical key assignment schemes in the computationally secure setting. Such a definition, called strong security with respect to keyindistinguishability (SKI-security), formalizes the requirement that the adversary is not able to learn any information about a key that it should not have access to, even if he has the additional capability of gaining access to encryption keys associated to all of the classes above the target class in the hierarchy. Notice that these encryption keys might leak through usage and their compromise could not directly lead to a compromise of the private information or the encryption key of the target class. Freire et al. [14] argued that their new notion is strictly stronger than the existing KI-security notion, and provide SKI-secure constructions for hierarchical key assignment schemes using pseudorandom functions and forward-secure pseudorandom generators. However, no formal security analysis was given to prove that SKI-security is actually stronger than traditional KI-security.

In this paper we focus on an information-theoretic approach which differs from the above computational approach since it does not depend on any computational assumption. Such an approach has already been considered in [13], [12] to analyze key assignment schemes for arbitrary access control policies, as well as in [4] to analyze time-bound hierarchical key assignment schemes. In such an unconditionally secure setting the security of a hierarchical key assign- ment scheme relies on the theoretical impossibility of breaking it, despite the computational power of an adversary coalition. In particular we prove that, in the unconditionally secure setting, SKI-security is not stronger than KI-security, i.e., the two definitions are, instead, fully equivalent from an informationtheoretic point of view.

The rest of this paper is organized as follows. In Section 2 we first recall the formal definition of hierarchical key assignment schemes and formalize the notions of KI-security and SKI-security from an information-theoretic point of view. Then, we prove in Section 3 the equivalence, in the unconditionally secure setting, of the two security notions. Finally, we draw our conclusions in Section 4

\section{HiERARCHICAL Key Assignment SCHEMES}

Consider a set of users divided into a number of disjoint classes, called security classes. A security class can represent a person, a department or a user group in an organization. A binary relation $\preceq$ that partially orders the set of classes $V$ is defined in accordance with authority, position or power of each class in $V$. The poset $(V, \preceq)$ is called a partially ordered hierarchy. For any two classes $u$ and $v$, the notation $u \preceq v$ is used to indicate that the users in $v$ can access $u$ 's data. We denote by $A_{v}$ the set $\{u \in V: u \preceq v\}$, for any $v \in V$, as shown in Figure 1 .

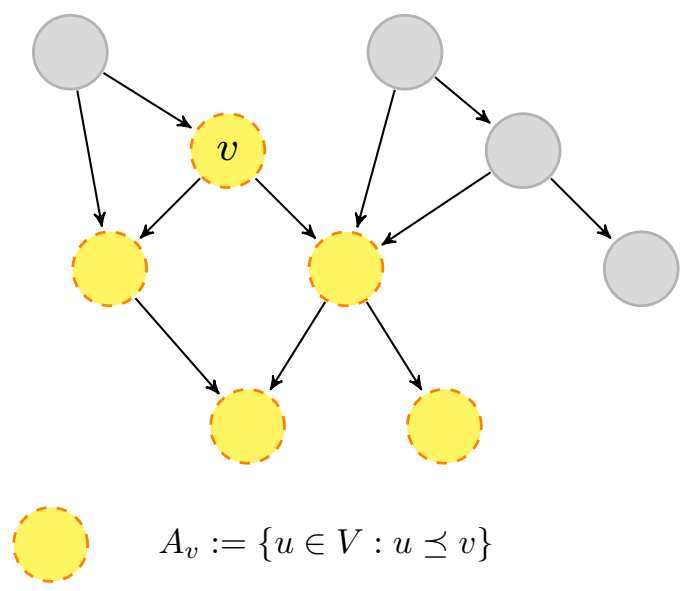

Fig. 1. An example of the $A_{v}$ set.

The partially ordered hierarchy $(V, \preceq)$ can be represented by a directed graph where each class corresponds to a vertex in the graph and there is an edge from class $v$ to class $u$ if and only if $u \preceq v$. Further, this graph can be simplified by eliminating all self-loops and edges which can be implied by the property of the transitive closure. We denote by $\mathcal{G}=(V, E)$ the resulting directed acyclic graph, which is called an access graph.

A hierarchical key assignment scheme for a partially ordered hierarchy represented by an access graph 
$\mathcal{G}=(V, E)$ is a method to assign a private information $s_{u}$ and a key $k_{u}$ to each class $u \in V$. The generation and distribution of the private information and keys is carried out by a trusted third party, the TA, which is connected to each class by means of a secure channel. The encryption key $k_{u}$ can be used by users belonging to class $u$ to protect their sensitive data by means of a symmetric crypto-system, whereas, the private information $s_{v}$ can be used by users belonging to class $v$ to compute the key $k_{u}$ for any class $u \in A_{v}$.

Following the line of [4], [12], [13], we formally define hierarchical key assignment schemes by using the entropy function (we refer the reader to the Appendix for some properties of the entropy function and to [7] for a complete treatment of Information Theory), mainly because this leads to a compact and simple description of the schemes and because the entropy approach takes into account all of the probability distributions on the keys assigned to the classes. The same approach has been used in [4], [12], [13] to analyze different kinds of key assignment schemes.

In the following, given a probability space $(\Omega, \mathcal{F}, \mathrm{P})$, we denote with a boldface capital letter a random variable defined on $\Omega$ (e.g., $\mathbf{X}: \Omega \rightarrow \mathbb{R}$ ) and taking values on a set, denoted by the corresponding capital letter (e.g., $X \subseteq \mathbb{R}$ ). The values such a random variable can take are denoted by the corresponding lower case letter (e.g., $x \in X$ ). Moreover, we write $p(x)$ for $p_{\mathbf{X}}(x):=\mathrm{P}\{\mathbf{X}=x\}_{x \in X}$ to refer to the distribution of $\mathbf{X}$. Hereafter all of the random variables considered will be discrete. Given a random variable $\mathbf{X}$, we denote by $\mathcal{H}(\mathbf{X})$ the Shannon entropy of $\mathbf{X}$.

Now we are ready to describe the correctness and security requirements that a hierarchical key assignment scheme has to satifsy.

Correctness Requirement: Each user can compute the key held by any class lower down in the hierarchy.

Formally, for each class $v \in V$ and each class $u \in A_{v}$, it holds that

$$
\mathcal{H}\left(\mathbf{K}_{u} \mid \mathbf{S}_{v}\right)=0 .
$$

Notice that the correctness requirement is equivalent to saying that the value of the private information $s_{v}$ held by each user belonging to a class $v \in V$ corresponds to a unique value of the key $k_{u}$, for each class $u \in A_{v}$.

Definition 2.1. Let $\mathcal{G}=(V, E)$ be an access graph. The set $\Sigma=\left\{\left(\mathbf{K}_{u}, \mathbf{S}_{u}\right)\right\}_{u \in V}$ is called a Hierarchical Key Assignment Scheme for $\mathcal{G}$ if the random variables $\mathbf{K}_{u}, \mathbf{S}_{u}$, for each $u \in V$, satisfy the above correctness requirement.

In order to achieve security, given a class $u \in V$, the key $k_{u}$ should be protected against attacks mounted by a coalition of users belonging to each class $v$ such that $u \notin A_{v}$. The amount of information available to the coalition strictly depends on the kind of security one would like to attain: first, we consider the case in which the coalition only owns the private information assigned to each class in the coalition; later, we will consider the case in which the coalition also holds the encryption keys assigned to classes which can access class $u$. Let $F_{u}:=\left\{v \in V: u \notin A_{v}\right\}$ be the set of classes which are not allowed to access the sensitive data of users in class $u$. Moreover, let $C_{u}:=\left\{v \in V: \quad u \in A_{v}\right\} \backslash\{u\}$ be the set of classes, different from $u$, which are entitled to access the sensitive data of users in class $u$. Figure 2 depicts the sets $F_{u}$ and $C_{u}$. Regarding the relationship among the sets $\{u\}, F_{u}$ and $C_{u}$, it can be easily verified that these sets are a partition of the set of nodes $V$.

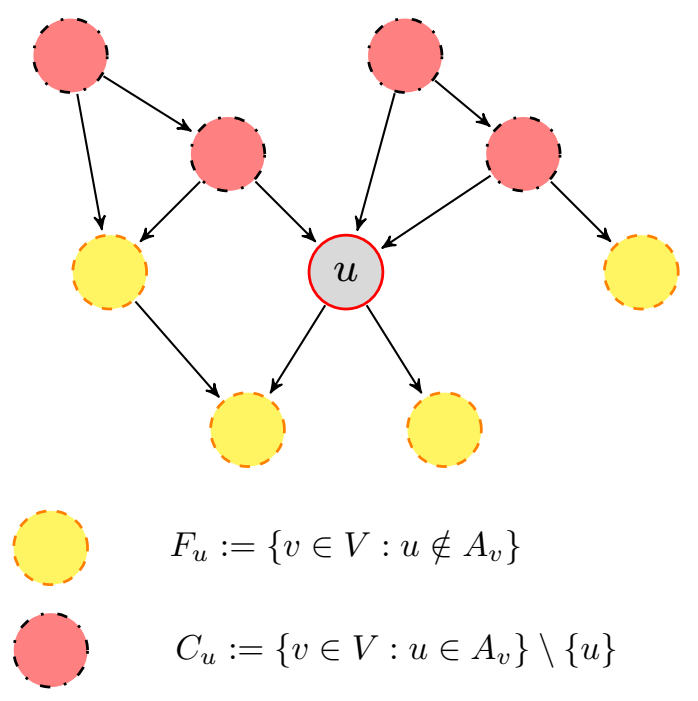

Fig. 2. An example of the $F_{u}$ and $C_{u}$ sets.

Given $u \in V$ and $X \subseteq F_{u}$, where $X=$ $\left\{v_{1}, v_{2}, \ldots, v_{n}\right\}$, let $\mathbf{S}_{X}$ be the random variable $\mathbf{S}_{v_{1}: v_{n}}=\left(\mathbf{S}_{v_{1}}, \mathbf{S}_{v_{2}}, \ldots, \mathbf{S}_{v_{n}}\right)$ whose law is the joint law of the random variables $\mathbf{S}_{v_{1}}, \mathbf{S}_{v_{2}}, \ldots, \mathbf{S}_{v_{n}}$. Similarly, given $Y \subseteq C_{u}$, where $Y=\left\{w_{1}, w_{2}, \ldots, w_{m}\right\}$, let $\mathbf{K}_{Y}$ be the random variable $\mathbf{K}_{w_{1}: w_{m}}=\left(\mathbf{K}_{w_{1}}, \mathbf{K}_{w_{2}}, \ldots, \mathbf{K}_{w_{m}}\right)$ whose law is the joint law of the random variables $\mathbf{K}_{w_{1}}, \mathbf{K}_{w_{2}}, \ldots, \mathbf{K}_{w_{m}}$.

We consider two different security notions: security with respect to key indistinguishability (KI-security) and strong security with respect to key indistinguishability (SKI-security). Security with respect to key indistinguishability formalizes the requirement that the adversary coalition is not able to learn any information about a key that it should not have access to, i.e., it is not able to distinguish it from a random string having the same length.

KI-Security Requirement: A coalition of users have absolutely no information about each key the coalition is not entitled to obtain.

Formally, for each class $u \in V$ and each coalition 
$X \subseteq F_{u}$, it holds that

$$
\mathcal{H}\left(\mathbf{K}_{u} \mid \mathbf{S}_{X}\right)=\mathcal{H}\left(\mathbf{K}_{u}\right) \text {. }
$$

Definition 2.2. Let $\mathcal{G}=(V, E)$ be an access graph and let $\Sigma=\left\{\left(\mathbf{K}_{u}, \mathbf{S}_{u}\right)\right\}_{u \in V}$ be a hierarchical key assignment scheme for $\mathcal{G}$. The scheme $\Sigma$ provides key indistinguishability (or KI-security) if the random variables $\mathbf{K}_{u}, \mathbf{S}_{u}$, for each $u \in V$, satisfy the above KI-security requirement.

On the other hand, strong security with respect to key indistinguishability formalizes the requirement that the adversary coalition is not able to learn any information about a key that it should not have access to, even though the coalition has the additional capability of gaining access to the encryption keys associated to other classes in the hierarchy.

SKI-Security Requirement: $A$ coalition of users have absolutely no information about each key the coalition is not entitled to obtain, even though the coalition has the additional capability of gaining access to the encryption keys associated to other classes in the hierarchy.

Formally, for each class $u \in V$, each coalition $X \subseteq F_{u}$, and each set $Y \subseteq C_{u}$, it holds that

$$
\mathcal{H}\left(\mathbf{K}_{u} \mid \mathbf{S}_{X}, \mathbf{K}_{Y}\right)=\mathcal{H}\left(\mathbf{K}_{u}\right) .
$$

Definition 2.3. Let $\mathcal{G}=(V, E)$ be an access graph and let $\Sigma=\left\{\left(\mathbf{K}_{u}, \mathbf{S}_{u}\right)\right\}_{u \in V}$ be a hierarchical key assignment scheme for $\mathcal{G}$. The scheme $\Sigma$ provides strong key indistinguishability (or SKI-security) if the random variables $\mathbf{K}_{u}, \mathbf{S}_{u}$, for each $u \in V$, satisfy the above SKI-security requirement.

Remark 2.1. It is evident that, given a scheme $\Sigma=\left\{\left(\mathbf{K}_{u}, \mathbf{S}_{u}\right)\right\}_{u \in V}$, in order to prove its KI-security it is enough proving that

$$
\forall u \in V, \quad \mathcal{H}\left(\mathbf{K}_{u} \mid \mathbf{S}_{F_{u}}\right)=\mathcal{H}\left(\mathbf{K}_{u}\right) .
$$

Eq. (1) and Lemma A.6 grant that the requirement also holds for all subsets $X \subseteq F_{u}$. Analogously, in order to prove SKI-security it is enough proving that

$$
\forall u \in V, \quad \mathcal{H}\left(\mathbf{K}_{u} \mid \mathbf{S}_{F_{u}}, \mathbf{K}_{C_{u}}\right)=\mathcal{H}\left(\mathbf{K}_{u}\right) .
$$

\section{EquiVALENCE OF SKI-SECURITY AND KI-SECURITY}

In the following we study the relationship between the notions of KI-security and SKI-security for hierarchical key assignment schemes. We first notice that SKI-security implies KI-security.

Indeed, for each $u \in V$, each $X \subseteq F_{u}$, and each $Y \subseteq C_{u}$, it follows from Lemma A.6 and A.1 that

$$
\mathcal{H}\left(\mathbf{K}_{u} \mid \mathbf{S}_{X}\right) \geq \mathcal{H}\left(\mathbf{K}_{u} \mid \mathbf{S}_{X}, \mathbf{K}_{Y}\right)=\mathcal{H}\left(\mathbf{K}_{u}\right) .
$$

Thus, if the SKI-security requirement holds, the KIsecurity requirement also holds.

We need the next definition.
Definition 3.1. Let $\mathcal{G}=(V, E)$ be an access graph and let $n \in \mathbb{N}$. The sequence $\left(u_{1}, u_{2}, \ldots, u_{n}\right) \in V^{n}$ is well ordered if $n=1$ or $n>1$ and for each $j \in\{2,3, \ldots, n\}$ it holds $\left\{u_{i}\right\}_{i=1}^{j-1} \subseteq F_{u_{j}}$.

We also recall the definition of topological sorting in a directed acyclic graph.

Definition 3.2. Let $\mathcal{G}=(V, E)$ be a directed acyclic graph, let $n \in \mathbb{N}, V=\left\{u_{i}\right\}_{i=1}^{n}$ and let $\sigma \in \mathcal{S}_{n}$ be a permutation. The sequence $X=\left(u_{\sigma(1)}, u_{\sigma(2)}, \ldots, u_{\sigma(n)}\right)$ is $a$ topological sort of $\mathcal{G}$ if for each $u, v \in V$ such that $(u, v) \in E$, then $u$ appears before $v$ in $X$.

Example 3.1. Let $\mathcal{G}=(V, E)$ be an access graph, let $n \in \mathbb{N}$ and let $X=\left(u_{n}, u_{n-1}, \ldots, u_{1}\right)$ be a topological sort of $\mathcal{G}$. It is easy to see that the sequence $\left(u_{1}, u_{2}, \ldots, u_{n}\right)$ is well ordered. Indeed, assume by contradiction that there exist $i, j \in\{1,2, \ldots, n\}$, with $i \leq j$, such that the users belonging to class $u_{i}$ are authorized to access the information of class $u_{j}$. Therefore we have that $\left(u_{i}, u_{j}\right) \in E$ and it follows that $u_{i}$ appears before $u_{j}$ in $X$, so that $i>j$, which is a contradiction.

The following lemma is crucial to understand the relationship between KI-security and SKI-security.

Lemma 3.1. Let $\mathcal{G}=(V, E)$ be an access graph, $\Sigma=\left\{\left(\mathbf{K}_{u}, \mathbf{S}_{u}\right)\right\}_{u \in V}$ be a KI-secure hierarchical key assignment scheme for $\mathcal{G}$ and let $n \in \mathbb{N}$. If $\left(u_{1}, u_{2}, \ldots, u_{n}\right) \in V^{n}$ is a well ordered sequence, then

$$
\mathcal{H}\left(\mathbf{K}_{u_{1}: u_{n}}\right)=\sum_{j=1}^{n} \mathcal{H}\left(\mathbf{K}_{u_{j}}\right) .
$$

In particular, the random variables $\mathbf{K}_{u_{1}}, \mathbf{K}_{u_{2}}, \ldots, \mathbf{K}_{u_{n}}$ are independent.

Proof: By Lemma A.2 it is enough proving that

$$
\mathcal{H}\left(\mathbf{K}_{u_{1}: u_{n}}\right) \geq \sum_{j=1}^{n} \mathcal{H}\left(\mathbf{K}_{u_{j}}\right) .
$$

Since $\left(u_{1}, u_{2}, \ldots, u_{n}\right) \in V^{n}$ is a well ordered sequence, for each $j \in\{2,3, \ldots, n\}$ it follows that $\left\{u_{i}\right\}_{i=1}^{j-1} \subseteq F_{u_{j}}$. Therefore, from the KI-security requirement we have that

$$
\mathcal{H}\left(\mathbf{K}_{u_{j}} \mid \mathbf{S}_{u_{1}: u_{j-1}}\right)=\mathcal{H}\left(\mathbf{K}_{u_{j}}\right) .
$$

Moreover, from the correctness requirement, it follows that

$$
\mathcal{H}\left(\mathbf{K}_{u_{j}} \mid \mathbf{S}_{u_{j}}\right)=0 .
$$

From Lemma A.3 it follows that

$$
\begin{aligned}
\mathcal{H}\left(\mathbf{K}_{u_{1}: u_{j-1}} \mid \mathbf{S}_{u_{1}: u_{j-1}}\right) & \leq \sum_{i=1}^{j-1} \mathcal{H}\left(\mathbf{K}_{u_{i}} \mid \mathbf{S}_{u_{1}: u_{j-1}}\right) \\
& \leq \sum_{i=1}^{j-1} \mathcal{H}\left(\mathbf{K}_{u_{i}} \mid \mathbf{S}_{u_{i}}\right)=0,
\end{aligned}
$$

thus we have 


$$
\mathcal{H}\left(\mathbf{K}_{u_{1}: u_{j-1}} \mid \mathbf{S}_{u_{1}: u_{j-1}}\right)=0 .
$$

Moreover, by Lemma A.6 it follows that

$$
\mathcal{H}\left(\mathbf{K}_{u_{1}: u_{j-1}} \mid \mathbf{S}_{u_{1}: u_{j-1}}, \mathbf{K}_{u_{j}}\right) \leq \mathcal{H}\left(\mathbf{K}_{u_{1}: u_{j-1}} \mid \mathbf{S}_{u_{1}: u_{j-1}}\right),
$$

therefore,

$$
\mathcal{H}\left(\mathbf{K}_{u_{1}: u_{j-1}} \mid \mathbf{S}_{u_{1}: u_{j-1}}, \mathbf{K}_{u_{j}}\right)=0 .
$$

Consider the conditional mutual information $\mathcal{I}\left(\mathbf{K}_{u_{j}} ; \mathbf{K}_{u_{1}: u_{j-1}} \mid \mathbf{S}_{u_{1}: u_{j-1}}\right)$. By using Lemma A.7 we deduce that

$$
\begin{aligned}
& \mathcal{H}\left(\mathbf{K}_{u_{j}} \mid \mathbf{S}_{u_{1}: u_{j-1}}\right)-\mathcal{H}\left(\mathbf{K}_{u_{j}} \mid \mathbf{K}_{u_{1}: u_{j-1}}, \mathbf{S}_{u_{1}: u_{j-1}}\right) \\
= & \mathcal{H}\left(\mathbf{K}_{u_{1}: u_{j-1}} \mid \mathbf{S}_{u_{1}: u_{j-1}}\right)-\mathcal{H}\left(\mathbf{K}_{u_{1}: u_{j-1}} \mid \mathbf{K}_{u_{j}}, \mathbf{S}_{u_{1}: u_{j-1}}\right),
\end{aligned}
$$

and, by using Equations (3) e (4), it follows that

$$
\mathcal{H}\left(\mathbf{K}_{u_{j}} \mid \mathbf{S}_{u_{1}: u_{j-1}}\right)=\mathcal{H}\left(\mathbf{K}_{u_{j}} \mid \mathbf{K}_{u_{1}: u_{j-1}}, \mathbf{S}_{u_{1}: u_{j-1}}\right) .
$$

Therefore, from Lemma A.4 we have that

$$
\begin{aligned}
\mathcal{H}\left(\mathbf{K}_{u_{1}: u_{n}}\right)= & \mathcal{H}\left(\mathbf{K}_{u_{1}}\right)+\sum_{j=2}^{n} \mathcal{H}\left(\mathbf{K}_{u_{j}} \mid \mathbf{K}_{u_{1}: u_{j-1}}\right) \\
\geq & \mathcal{H}\left(\mathbf{K}_{u_{1}}\right) \\
& +\sum_{j=2}^{n} \mathcal{H}\left(\mathbf{K}_{u_{j}} \mid \mathbf{K}_{u_{1}: u_{j-1}}, \mathbf{S}_{u_{1}: u_{j-1}}\right) \\
= & \mathcal{H}\left(\mathbf{K}_{u_{1}}\right)+\sum_{j=2}^{n} \mathcal{H}\left(\mathbf{K}_{u_{j}} \mid \mathbf{S}_{u_{1}: u_{j-1}}\right) \\
= & \mathcal{H}\left(\mathbf{K}_{u_{1}}\right)+\sum_{j=2}^{n} \mathcal{H}\left(\mathbf{K}_{u_{j}}\right) \\
= & \sum_{j=1}^{n} \mathcal{H}\left(\mathbf{K}_{u_{j}}\right) .
\end{aligned}
$$

Moreover, from Lemma A.2, it holds that the random variables $\mathbf{K}_{u_{1}}, \mathbf{K}_{u_{2}}, \ldots, \mathbf{K}_{u_{n}}$ are independent. Thus, the lemma holds.

We are going to show that such a result allows proving the equivalence of the two security notions. Moreover, it is worth noting here that, reasoning as before, we can prove that KI-security is a sufficient condition for the independence of all of the keys in a hierarchical key assignment scheme. Indeed, the following theorem holds.

Theorem 3.1. Let $\mathcal{G}=(V, E)$ be an access graph and let $\Sigma=\left\{\left(\mathbf{K}_{u}, \mathbf{S}_{u}\right)\right\}_{u \in V}$ be a hierarchical key assignment scheme for $\mathcal{G}$. If $\Sigma$ is KI-secure for $\mathcal{G}$, then the random variables $\left\{\mathbf{K}_{u}\right\}_{u \in V}$ are independent.

Proof: It suffices proceeding as in Lemma 3.1 using the well ordered sequence resulting by any arbitrary topological sort of $\mathcal{G}$ reverse ordered (see for instance Example 3.1).
As said before, Lemma 3.1 will be used to prove the equivalence of KI-security and SKI-security. However, before proving the equivalence, we need some additional preliminary results.

Lemma 3.2. Let $\mathcal{G}=(V, E)$ be an access graph, let $\Sigma=\left\{\left(\mathbf{K}_{u}, \mathbf{S}_{u}\right)\right\}_{u \in V}$ be a KI-secure hierarchical key assignment scheme for $\mathcal{G}$ and let $n, m \in \mathbb{N}$. If $\left(u_{1}, u_{2}, \ldots, u_{n+m}\right) \in V^{n+m}$ is a well ordered sequence, then

$$
\mathcal{H}\left(\mathbf{K}_{u_{n+1}: u_{n+m}} \mid \mathbf{K}_{u_{n}}, \mathbf{S}_{u_{1}: u_{n-1}}\right)=\sum_{j=1}^{m} \mathcal{H}\left(\mathbf{K}_{u_{n+j}}\right) .
$$

Proof: By Lemma A.1 and A.2 it is enough showing that

$$
\mathcal{H}\left(\mathbf{K}_{u_{n+1}: u_{n+m}} \mid \mathbf{K}_{u_{n}}, \mathbf{S}_{u_{1}: u_{n-1}}\right) \geq \sum_{j=1}^{m} \mathcal{H}\left(\mathbf{K}_{u_{n+j}}\right) .
$$

From the correctness requirement it follows that, for each $j \in\{2,3, \ldots, m+1\}$,

$$
\begin{aligned}
\mathcal{H}\left(\mathbf{K}_{u_{n}: u_{n+j-1}} \mid \mathbf{S}_{u_{1}: u_{n+j-1}}\right) & \leq \sum_{i=0}^{j-1} \mathcal{H}\left(\mathbf{K}_{u_{n+i}} \mid \mathbf{S}_{u_{1}: u_{n+j-1}}\right) \\
& \leq \sum_{i=0}^{j-1} \mathcal{H}\left(\mathbf{K}_{u_{n+i}} \mid \mathbf{S}_{u_{n+i}}\right)=0,
\end{aligned}
$$

thus

$$
\mathcal{H}\left(\mathbf{K}_{u_{n}: u_{n+j-1}} \mid \mathbf{S}_{u_{1}: u_{n+j-1}}\right)=0,
$$

and consequently,

$$
\mathcal{H}\left(\mathbf{K}_{u_{n}: u_{n+j-1}} \mid \mathbf{K}_{u_{n+j}}, \mathbf{S}_{u_{1}: u_{n+j-1}}\right)=0 .
$$

Consider the conditional mutual information $\mathcal{I}\left(\mathbf{K}_{u_{n+j}} ; \mathbf{K}_{u_{n}: u_{n+j-1}} \mid \mathbf{S}_{u_{1}: u_{n+j-1}}\right)$. We have that

$$
\begin{aligned}
& \mathcal{H}\left(\mathbf{K}_{u_{n+j}} \mid \mathbf{S}_{u_{1}: u_{n+j-1}}\right) \\
& -\mathcal{H}\left(\mathbf{K}_{u_{n+j}} \mid \mathbf{K}_{u_{n}: u_{n+j-1}}, \mathbf{S}_{u_{1}: u_{n+j-1}}\right) \\
= & \mathcal{H}\left(\mathbf{K}_{u_{n}: u_{n+j-1}} \mid \mathbf{S}_{u_{1}: u_{n+j-1}}\right) \\
& -\mathcal{H}\left(\mathbf{K}_{u_{n}: u_{n+j-1}} \mid \mathbf{K}_{u_{n+j}}, \mathbf{S}_{u_{1}: u_{n+j-1}}\right),
\end{aligned}
$$

and, by using (5) and (6), it follows that

$\mathcal{H}\left(\mathbf{K}_{u_{n+j}} \mid \mathbf{S}_{u_{1}: u_{n+j-1}}\right)=\mathcal{H}\left(\mathbf{K}_{u_{n+j}} \mid \mathbf{K}_{u_{n}: u_{n+j-1}}, \mathbf{S}_{u_{1}: u_{n+j-1}}\right)$.

Now, letting $Q=\mathcal{H}\left(\mathbf{K}_{u_{n+1}: u_{n+m}} \mid \mathbf{K}_{u_{n}}, \mathbf{S}_{u_{1}: u_{n-1}}\right)$, from Lemma A.5 we have that 


$$
\begin{aligned}
Q= & \mathcal{H}\left(\mathbf{K}_{u_{n+1}} \mid \mathbf{K}_{u_{n}}, \mathbf{S}_{u_{1}: u_{n-1}}\right) \\
& +\sum_{j=2}^{m} \mathcal{H}\left(\mathbf{K}_{u_{n+j}} \mid \mathbf{K}_{u_{n}: u_{n+j-1}}, \mathbf{S}_{u_{1}: u_{n-1}}\right) \\
\geq & \mathcal{H}\left(\mathbf{K}_{u_{n+1}} \mid \mathbf{K}_{u_{n}}, \mathbf{S}_{u_{1}: u_{n}}\right) \\
& +\sum_{j=2}^{m} \mathcal{H}\left(\mathbf{K}_{u_{n+j}} \mid \mathbf{K}_{u_{n}: u_{n+j-1}}, \mathbf{S}_{u_{1}: u_{n+j}-1}\right) \\
= & \mathcal{H}\left(\mathbf{K}_{u_{n+1}} \mid \mathbf{S}_{u_{1}: u_{n}}\right) \\
& +\sum_{j=2}^{m} \mathcal{H}\left(\mathbf{K}_{u_{n+j}} \mid \mathbf{S}_{u_{1}: u_{n+j-1}}\right) \\
= & \sum_{j=1}^{m} \mathcal{H}\left(\mathbf{K}_{u_{n+j}}\right)
\end{aligned}
$$

in which the last equality follows from the well ordering assumption and from the KI-security requirement. Thus, the lemma holds.

Corollary 3.1. Let $\mathcal{G}=(V, E)$ be an access graph, let $\Sigma=\left\{\left(\mathbf{K}_{u}, \mathbf{S}_{u}\right)\right\}_{u \in V}$ be a KI-secure hierarchical key assignment scheme for $\mathcal{G}$ and let $n, m \in \mathbb{N}$. If $\left(u_{1}, u_{2}, \ldots, u_{n+m}\right) \in V^{n+m}$ is a well ordered sequence, then

$$
\mathcal{H}\left(\mathbf{K}_{u_{n+1}: u_{n+m}} \mid \mathbf{S}_{u_{1}: u_{n-1}}\right)=\sum_{j=1}^{m} \mathcal{H}\left(\mathbf{K}_{u_{n+j}}\right) .
$$

Proof: From Lemma A.6 and 3.1 it follows that

$$
\begin{aligned}
\mathcal{H}\left(\mathbf{K}_{u_{n+1}: u_{n+m}} \mid \mathbf{K}_{u_{n}}, \mathbf{S}_{u_{1}: u_{n-1}}\right) & \leq \mathcal{H}\left(\mathbf{K}_{u_{n+1}: u_{n+m}} \mid \mathbf{S}_{u_{1}: u_{n-1}}\right) \text { i.e., } \\
& \leq \mathcal{H}\left(\mathbf{K}_{u_{n+1}: u_{n+m}}\right) \\
& =\sum_{j=1}^{m} \mathcal{H}\left(\mathbf{K}_{u_{n+j}}\right), \quad \text { Thus, }
\end{aligned}
$$

consequently, the thesis follows from Lemma 3.2

Theorem 3.2. Let $\mathcal{G}=(V, E)$ be an access graph, $\Sigma=\left\{\left(\mathbf{K}_{u}, \mathbf{S}_{u}\right)\right\}_{u \in V}$ a KI-secure hierarchical key assignment scheme for $\mathcal{G}, n, m \in \mathbb{N}$. If $\left(u_{1}, u_{2}, \ldots, u_{n+m}\right) \in V^{n+m} s$ a well ordered sequence, then

$$
\mathcal{H}\left(\mathbf{K}_{u_{n}} \mid \mathbf{K}_{u_{n+1}: u_{n+m}}, \mathbf{S}_{u_{1}: u_{n-1}}\right)=\mathcal{H}\left(\mathbf{K}_{u_{n}}\right) .
$$

Proof: Consider the conditional mutual information $\mathcal{I}\left(\mathbf{K}_{u_{n}} ; \mathbf{K}_{u_{n+1}: u_{n+m}} \mid \mathbf{S}_{u_{1}: u_{n-1}}\right)$. It holds that

$$
\begin{aligned}
& \mathcal{H}\left(\mathbf{K}_{u_{n}} \mid \mathbf{S}_{u_{1}: u_{n-1}}\right)-\mathcal{H}\left(\mathbf{K}_{u_{n}} \mid \mathbf{K}_{u_{n+1}: u_{n+m}}, \mathbf{S}_{u_{1}: u_{n-1}}\right) \\
= & \mathcal{H}\left(\mathbf{K}_{u_{n+1}: u_{n+m}} \mid \mathbf{S}_{u_{1}: u_{n-1}}\right) \\
& -\mathcal{H}\left(\mathbf{K}_{u_{n+1}: u_{n+m}} \mid \mathbf{K}_{u_{n}}, \mathbf{S}_{u_{1}: u_{n-1}}\right) \\
= & \sum_{j=1}^{m} \mathcal{H}\left(\mathbf{K}_{u_{n+j}}\right)-\sum_{j=1}^{m} \mathcal{H}\left(\mathbf{K}_{u_{n+j}}\right) \\
= & 0
\end{aligned}
$$

where in Eq. (7) we used Corollary 3.1 and Lemma 3.2. It holds that

$$
\begin{aligned}
\mathcal{H}\left(\mathbf{K}_{u_{n}} \mid \mathbf{K}_{u_{n+1}: u_{n+m}}, \mathbf{S}_{u_{1}: u_{n-1}}\right) & =\mathcal{H}\left(\mathbf{K}_{u_{n}} \mid \mathbf{S}_{u_{1}: u_{n-1}}\right) \\
& =\mathcal{H}\left(\mathbf{K}_{u_{n}}\right),
\end{aligned}
$$

where in the last equality we used the KI-security requirement. Thus, the theorem holds.

We are now in the position to state the following theorem, which is the main result and contribution of this paper.

Theorem 3.3. Let $\mathcal{G}=(V, E)$ be an access graph and let $\Sigma=\left\{\left(\mathbf{K}_{u}, \mathbf{S}_{u}\right)\right\}_{u \in V}$ be a hierarchical key assignment scheme for $\mathcal{G}$. If $\Sigma$ is KI-secure for $\mathcal{G}$, then $\Sigma$ is also SKIsecure for $\mathcal{G}$.

Proof: Let $u \in V$. By Remark 2.1, the SKI-security of $\Sigma$ is proved if we can show that Eq. (2) holds, i.e.,

$$
\mathcal{H}\left(\mathbf{K}_{u} \mid \mathbf{K}_{C_{u}}, \mathbf{S}_{F_{u}}\right)=\mathcal{H}\left(\mathbf{K}_{u}\right) .
$$

Let $n, m \in \mathbb{N}$ and $u_{1}, u_{2}, \ldots, u_{n+m}$ be an enumeration of $V$ such that $\left\{u_{i}\right\}_{i=1}^{n-1}=F_{u}$, $u_{n}=u, \quad$ and $\quad\left\{u_{i}\right\}_{i=n+1}^{n+m}=C_{u}$. Moreover, let $\sigma \in \mathcal{S}_{n-1}$ and $\tau \in \mathcal{S}_{m}$ be permutations such that $\left(u_{\sigma(1)}, u_{\sigma(2)}, \ldots, u_{\sigma(n-1)}\right)$ is a topological sort of $F_{u}$ and $\left(u_{\tau(n+1)}, u_{\tau(n+2)}, \ldots, u_{\tau(n+m)}\right)$ a topological sort of $C_{u}$. Then, the sequence

$\left(u_{\sigma(n-1)}, \ldots, u_{\sigma(2)}, u_{\sigma(1)}, u_{n}, u_{\tau(n+m)}, \ldots, u_{\tau(n+2)}, u_{\tau(n+1)}\right)$

is well ordered, thus, by Theorem 3.2 it follows that

$$
\mathcal{H}\left(\mathbf{K}_{u_{n}} \mid \mathbf{K}_{u_{\tau(n+m)}: u_{\tau(n+1)}}, \mathbf{S}_{u_{\sigma(n-1)}: u_{\sigma(1)}}\right)=\mathcal{H}\left(\mathbf{K}_{u_{n}}\right),
$$$$
\mathcal{H}\left(\mathbf{K}_{u} \mid \mathbf{K}_{C_{u}}, \mathbf{S}_{F_{u}}\right)=\mathcal{H}\left(\mathbf{K}_{u}\right) .
$$

Thus, the theorem holds.

\section{Conclusions}

Freire, Paterson and Poettering [14] recently proposed a new security notion for hierarchical key assignment schemes in the computationally secure setting, called strong security with respect to key indistinguishability (SKI-security). They argued that their new notion is stronger than the traditional KI-security one, but did not prove formally that indeed this is the case. In this paper we showed that, in the unconditionally secure setting, SKI-security is not stronger than KI-security, i.e., the two definitions are fully equivalent from an information-theoretic point of view.

\section{APpendix A}

We recall here a few basic results of Information Theory used in our definitions and proofs. For a complete treatment of the subject the reader is advised to consult [7]. 
Definition A.1. Let $\mathbf{X}$ be a random variable with distribution $p(x)$. The entropy of $\mathbf{X}$ is the operator

$$
\mathcal{H}(\mathbf{X}):=-\sum_{x \in X} p(x) \log (p(x))
$$

where log denotes base 2 logarithm.

The entropy satisfies the following property

$$
\mathcal{H}(\mathbf{X}) \geq 0
$$

with equality if and only if there exists $x \in X$ such that $p(x)=1$.

Definition A.2. Let $\mathbf{X}, \mathbf{Y}$ be random variables with joint distribution $p(x, y)$. The joint entropy of $\mathbf{X}$ and $\mathbf{Y}$ is the operator

$$
\mathcal{H}(\mathbf{X}, \mathbf{Y}):=-\sum_{x \in X} \sum_{y \in Y} p(x, y) \log (p(x, y)) .
$$

Definition A.3. Let $\mathbf{X}, \mathbf{Y}$ be random variables with joint distribution $p(x, y)$ and conditional distribution $p(x \mid y)$. The conditional entropy of $\mathbf{X}$ given $\mathbf{Y}$ is the operator

$$
\mathcal{H}(\mathbf{X} \mid \mathbf{Y}):=-\sum_{x \in X} \sum_{y \in Y} p(x, y) \log (p(x \mid y)) .
$$

Lemma A.1. Let $\mathbf{X}, \mathbf{Y}$ be random variables. Then,

$$
\mathcal{H}(\mathbf{X} \mid \mathbf{Y}) \leq \mathcal{H}(\mathbf{X}) .
$$

Moreover, the equality holds if and only if $\mathbf{X} e \mathbf{Y}$ are independent.

Lemma A.2. Let $n \in \mathbb{N}$ and let $\mathbf{X}_{1}, \mathbf{X}_{2}, \ldots, \mathbf{X}_{n}$ be random variables. It holds that

$$
\mathcal{H}\left(\mathbf{X}_{1: n}\right) \leq \sum_{i=1}^{n} \mathcal{H}\left(\mathbf{X}_{i}\right)
$$

Moreover, the equality holds if and only if $\mathbf{X}_{1}, \mathbf{X}_{2}, \ldots, \mathbf{X}_{n}$ are independent.

Lemma A.3. Let $n \in \mathbb{N}$ and let $\mathbf{X}_{1}, \mathbf{X}_{2}, \ldots, \mathbf{X}_{n}, \mathbf{Y}$ be random variables. It holds that

$$
\mathcal{H}\left(\mathbf{X}_{1: n} \mid \mathbf{Y}\right) \leq \sum_{i=1}^{n} \mathcal{H}\left(\mathbf{X}_{i} \mid \mathbf{Y}\right)
$$

Lemma A.4. Let $n \in \mathbb{N} \backslash\{1\}$ and let $\mathbf{X}_{1}, \mathbf{X}_{2}, \ldots, \mathbf{X}_{n}$ be random variables. It holds that

$$
\mathcal{H}\left(\mathbf{X}_{1, n}\right)=\mathcal{H}\left(\mathbf{X}_{1}\right)+\sum_{i=2}^{n} \mathcal{H}\left(\mathbf{X}_{i} \mid \mathbf{X}_{1:(i-1)}\right) .
$$

Lemma A.5. Let $n \in \mathbb{N} \backslash\{1\}$ and let $\mathbf{X}_{1}, \mathbf{X}_{2}, \ldots, \mathbf{X}_{n}, \mathbf{Y}$ be random variables. It holds that

$$
\mathcal{H}\left(\mathbf{X}_{1: n} \mid \mathbf{Y}\right)=\mathcal{H}\left(\mathbf{X}_{1} \mid \mathbf{Y}\right)+\sum_{i=2}^{n} \mathcal{H}\left(\mathbf{X}_{i} \mid \mathbf{X}_{1:(i-1)}, \mathbf{Y}\right)
$$

Definition A.4. Let $\mathbf{X}, \mathbf{Y}$ be random variables. The $\mathrm{mu}-$ tual information between $\mathbf{X}$ and $\mathbf{Y}$ is the operator

$$
\mathcal{I}(\mathbf{X} ; \mathbf{Y}):=\mathcal{H}(\mathbf{X})-\mathcal{H}(\mathbf{X} \mid \mathbf{Y}) .
$$

From Lemma A.1 follows that $\mathcal{I}(\mathbf{X} ; \mathbf{Y}) \geq 0$ and it is easy to verify that $\mathcal{I}(\mathbf{X} ; \mathbf{Y})=\mathcal{I}(\mathbf{Y} ; \mathbf{X})$.

Definition A.5. Let $\mathbf{X}, \mathbf{Y}, \mathbf{Z}$ be random variables. The conditional mutual information of $\mathbf{X}$ and $\mathbf{Y}$ given $\mathbf{Z}$ is the operator

$$
\mathcal{I}(\mathbf{X} ; \mathbf{Y} \mid \mathbf{Z}):=\mathcal{H}(\mathbf{X} \mid \mathbf{Z})-\mathcal{H}(\mathbf{X} \mid \mathbf{Y}, \mathbf{Z}) .
$$

Lemma A.6. Let $\mathbf{X}, \mathbf{Y}, \mathbf{Z}$ be random variables. It holds that $\mathcal{I}(\mathbf{X} ; \mathbf{Y} \mid \mathbf{Z}) \geq 0$. Moreover,

$$
\mathcal{H}(\mathbf{X} \mid \mathbf{Y}, \mathbf{Z}) \leq \mathcal{H}(\mathbf{X} \mid \mathbf{Z}) .
$$

Lemma A.7. Let $\mathbf{X}, \mathbf{Y}, \mathbf{Z}$ be random variables. It holds that

$$
\mathcal{I}(\mathbf{X} ; \mathbf{Y} \mid \mathbf{Z})=\mathcal{I}(\mathbf{Y} ; \mathbf{X} \mid \mathbf{Z}) .
$$

In particular,

$$
\mathcal{H}(\mathbf{X} \mid \mathbf{Z})-\mathcal{H}(\mathbf{X} \mid \mathbf{Y}, \mathbf{Z})=\mathcal{H}(\mathbf{Y} \mid \mathbf{Z})-\mathcal{H}(\mathbf{Y} \mid \mathbf{X}, \mathbf{Z}) .
$$

\section{References}

[1] S. G. Akl and P. D. Taylor. Cryptographic Solution to a Problem of Access Control in a Hierarchy. ACM Transactions on Computer Systems, 1(3):239-248, 1983.

[2] M. J. Atallah, M. Blanton, N. Fazio, and K. B. Frikken. Dynamic and Efficient Key Management for Access Hierarchies. ACM Trans. Inf. Syst. Secur., 12(3), 2009. Preliminary version in Proc. of the ACM Conference on Computer and Communications Security 2006.

[3] G. Ateniese, A. De Santis, A. L. Ferrara, and B. Masucci. Provably-Secure Time-Bound Hierarchical Key Assignment Schemes. Journal of Cryptology, 25(2):243-270, 2012.

[4] G. Ateniese, A. De Santis, A. L. Ferrara, and B. Masucci. A Note on Time-Bound Hierarchical Key Assignment Schemes. Information Processing Letters, 113(5-6):151-155, 2013.

[5] T. Chen and Y. Chung. Hierarchical Access Control based on Chinese Remainder Theorem and Symmetric Algorithm. Computers \& Security, 21(6):565-570, 2002.

[6] H.-Y. Chien. Efficient Time-Bound Hierarchical Key Assignment Scheme. IEEE Transaction on Knowledge and Data Engineering, 16(10):1301-1304, 2004.

[7] T. M. Cover and J. A. Thomas. Elements of Information Theory (Wiley Series in Telecommunications and Signal Processing). WileyInterscience, 2006

[8] J. Crampton, K. Martin, and P. Wild. On Key Assignment for Hierarchical Access Control. In Proc. of the 19th IEEE Computer Security Foundations Workshop, pages 98-111, 2006.

[9] P. D'Arco, A. D. Santis, A. L. Ferrara, and B. Masucci. Variations on a Theme by Akl and Taylor: Security and Tradeoffs. Theoretical Computer Science, 411(1):213-227, 2010.

[10] A. De Santis, A. L. Ferrara, and B. Masucci. Cryptographic Key Assignment Schemes for any Access Control Policy. Information Processing Letters, 92(4):199-205, 2004.

[11] A. De Santis, A. L. Ferrara, and B. Masucci. Enforcing the Security of a Time-Bound Hierarchical Key Assignment Scheme. Information Sciences, 176(12):1684-1694, 2006.

[12] A. De Santis, A. L. Ferrara, and B. Masucci. Unconditionally Secure Key Assignment Schemes. Discrete Applied Mathematics, 154(2):234-252, 2006.

[13] A. L. Ferrara and B. Masucci. An Information- TheoreticApproach to the Access Control Problem. In ICTCS, pages 342-354, 2003.

[14] E. S. V. Freire, K. G. Paterson, and B. Poettering. Simple, Efficient and Strongly KI-Secure Hierarchical Key Assignment Schemes. In Proceedings of the 13th International Conference on Topics in Cryptology, CT-RSA'13, pages 101-114, Berlin, Heidelberg, 2013. Springer-Verlag.

[15] L. Harn and H. Y. Lin. A Cryptographic Key Generation Scheme for Multilevel Data Security. Computers \& Security, 9(6):539-546, 1990. 
[16] H. F. Huang and C. C. Chang. A New Cryptographic Key Assignment Scheme with Time-Constraint Access Control in a Hierarchy. Computer Standards E Interfaces, 26:159-166, 2004.

[17] M. S. Hwang. A Cryptographic Key Assignment Scheme in a Hierarchy for Access Control. Mathematical and Computational Modeling, 26(1):27-31, 1997.

[18] H. T. Liaw, S. J. Wang, and C. L. Lei. A Dynamic Cryptographic Key Assignment Scheme in a Tree Structure. Computers and Mathematics with Applications, 25(6):109-114, 1993.

[19] C. H. Lin. Dynamic Key Management Schemes for Access Control in a Hierarchy. Computer Communications, 20:13811385, 1997.

[20] I. C. Lin, M. S. Hwang, and C. C. Chang. A New Key Assignment Scheme for Enforcing Complicated Access Control Policies in Hierarchy. Future Generation Computer Systems, 19:157-462, 2003.

[21] S. J. MacKinnon, P. D. Taylor, H. Meijer, and S. G. Akl. An Optimal Algorithm for Assigning Cryptographic Keys to Control Access in a Hierarchy. IEEE Transaction on Computers, 34(9):797-802, 1985.

[22] R. S. Sandhu. Cryptographic Implementation of a Tree Hierarchy for Access Control. Information Processing Letters, 27(2):9598, 1988.

[23] V. Shen and T. Chen. A Novel Key Management Scheme based on Discrete Logarithms and Polynomial Interpolations. Computers E Security, 21(2):164-171, 2002.

[24] Q. Tang and C. J. Mitchell. Comments on a Cryptographic Key Assignment Scheme. Computer Standards \& Interfaces, 27:323326, 2005.

[25] W.-G. Tzeng. A Time-Bound Cryptographic Key Assignment Scheme for Access Control in a Hierarchy. IEEE Transactions on Knowledge and Data Engineering, 14(1):182-188, 2002.

[26] W.-G. Tzeng. A Secure System for Data Access Based on Anonymous and Time-Dependent Hierarchical Keys. In Proc. of the ACM Symposium on Information, Computer and Communications Security, pages 223-230, 2006.

[27] S.-Y. Wang and C.-Laih. Merging: an Efficient Solution for a Time-Bound Hierarchical Key Assignment Scheme. IEEE Transactions on Dependable and Secure Computing, 3(1):91-100, 2006.

[28] J. Yeh. An RSA-Based Time-Bound Hierarchical Key Assignment Scheme for Electronic Article Subscription. In Proc. of the ACM CIKM International Conference on Information and Knowledge Management, pages 285-286, 2005.

[29] J. Yeh, R. Chow, and R. Newman. A Key Assignment for Enforcing Access Control Policy Exceptions. In Proc. of the International Symposium on Internet Technology, pages 54-59, 1998

[30] X. Yi. Security of Chien's Efficient Time-Bound Hierarchical Key Assignment Scheme. IEEE Transactions on Knowledge and Data Engineering, 17(9):1298-1299, 2005.

[31] X. Yi and Y. Ye. Security of Tzeng's Time-Bound Key Assignment Scheme for Access Control in a Hierarchy. IEEE Transactions on Knowledge and Data Engineering, 15(4):10541055,2003

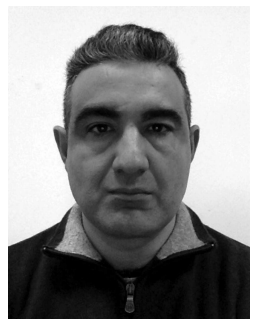

Massimo Cafaro is an Assistant Professor at the Department of Innovation Engineering of the University of Salento. His research covers High Performance, Distributed and Cloud/Grid Computing, security and cryptography. He received a degree in Computer Science from the University of Salerno and a Ph.D. in Computer Science from the University of Bari. He is a Senior Member of IEEE and of IEEE Computer Society, and Senior Member ACM. He authored or coauthored more than 90 refereed papers on parallel, distributed and $\mathrm{grid} /$ cloud computing. He co-authored and holds a patent on distributed database technologies. His research interests are focused on both theoretical and practical aspects of parallel and distributed computing, security and cryptography, with particular attention to the design and analysis of algorithms.

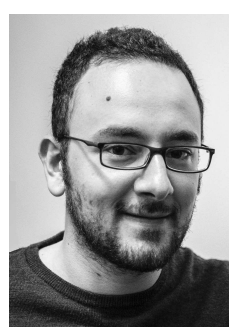

Roberto Civino received the M.Sc. degree in Mathematics (cum laude) from the University of Salento in 2014. His interests are in the field of security and cryptography.

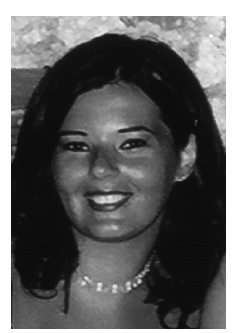

Barbara Masucci received the Laurea degree in Computer Science (cum laude) from the University of Salerno in 1996. From September 1999 to April 2000 she was a Visiting Researcher at the Centre for Applied Cryptographic Research, in the Department of Combinatorics and Optimization of the University of Waterloo, Ontario, Canada. In 2001 she received a Ph.D. in Computer Science from the University of Salerno. From March 2001 to February 2002 she was a Post-Doctoral Fellow at the Dipartimento di Informatica ed Applicazioni, University of Salerno. In July 2001 she visited the Departament de Matemática Aplicada IV at the Universitat Politécnica de Catalunya, Barcelona, Spain. Since March 2002 she has been an Assistant Professor at the Dipartimento di Informatica, University of Salerno. Her research interests include Algorithms, Data Security, and Cryptography. In particular, she has been working on the design and analysis of secure and efficient cryptographic protocols. 